\title{
КОГНИТИВНЫЙ ПОТЕНЦИАЛ ПРЕДЛОЖЕНИЙ С ГЕНЕРАЛИЗОВАННЫМ СУБЬЕКТОМ В АНГЛОЯЗЫЧНОМ ПУБЛИЦИСТИЧЕСКОМ ДИСКУРСЕ
}

\author{
Н. В. Ильичева \\ Воронежский государственный университет \\ COGNITIVE POTENTIAL OF SENTENCES WITH \\ A GENERALIZED SUBJECT IN ENGLISH JOURNALISTIC DISCOURSE
}

\author{
N. V. Ilyicheva \\ Voronezh State University
}

\begin{abstract}
Аннотация: в статье рассматриваются вопросы когнитивного потенщиала предложений с генерализованным субъектом. Такой тип предложений характеризуется определенным набором формальных маркеров, которые заимствованы ими у других грамматических категорий. С точки зрения семантики субъект генерализованных предложений обозначает любое лицо или предмет, который обобщает и репрезентует всех и каждого члена сообщества в ситуации временной нелокализованности. Прагматическая установка высказываний с генерализованным субъектом соответствует двум основным иелям публицистического дискурса: информирование и влияние. В исследовании приводятся результаты анализа использования и прагматической направленности данного вида предложений в текстах публицистического дискурса. Генерализованные предложения, характеризующие все субъекты множества, а также употребляющиеся в ситуачии заключения или вывода, используются в публицистичеком дискурсе для информирования адресата. В то же время предложения с генерализованным субъектом являются сильным средством воздействия на адресата, выражая эмоциионально-модальную окраску и представляя ссылку на авторитетные источники. Влияние на слушающего или читающего является значительным, поскольку важность действия подчеркивается семой распространения на всех возможных адресатов и необходимостью действия по примеру всех и вместе со всеми.

Ключевые слова: предложения с генерализованным субъектом, значение обобщенности, публицистический дискурс, прагматика дискурса.
\end{abstract}

\begin{abstract}
Such sentences are characterized by a certain number of formal markers borrowed from other grammatical categories. From the point of view of semantics, the subject of generalized sentences represents any person or object that generalizes and represents each and every member of its class in the situation of temporary non-localization. The pragmatic goal of statements with a generalized subject corresponds to the two main goals of journalistic discourse. They are to inform the readers and to influence them. The study shows the results of the analysis of the usage and pragmatic goals of sentences with a generalized subject in journalistic discourse. Sentences of this type are used to characterize all the members of a set and to make a conclusion, which helps them to inform the addressee. At the same time, sentences with a generalized subject are an efficient way of influencing the addressee by means of expressing the emotional attitude of the author and presenting a reference to authoritative sources. In this case the influence on the listener or reader is significant, since the importance of the action is emphasized by the reference to all possible addressees, and the necessity to follow everyone's example.
\end{abstract}

Key words: sentences with a generalized subject, the meaning of generality, journalistic discourse, discourse pragmatics.

(с) Ильичева Н. В., 2021

Контент доступен под лицензией Creative Commons Attribution 4.0 License.

The content is available under Creative Commons Attribution 4.0 License. 


\section{Введение}

Когнитивная лингвистика, изучающая «ментальные» основы продуцирования речи и восприятия языковых явлений, репрезентацию языковых структур в речи и их связь с нашим осознанием реальной действительности, дает возможность понять связь «ментальных репрезентаций явлений действительности и их языковых “привязок” (коррелятивных им языковых форм)» [1]. Цель когнитивной лингвистики - «понять, как осуществляются процессы восприятия, категоризации, классификации мира и осмысления мира, как происходит накопление знаний, какие системы обеспечивают различные виды деятельности с информацией» [2, с. 12].

Когнитивные операции, выполняемые в процессе коммуникации на английском языке, многообразны и важную роль среди них играет обобщение, или генерализация, которая в языковом сознании носителей языка является одной из форм представления знания путем переходов «частное- общее» / «общеечастное» и характеризуется высоким уровнем абстракции.

Генерализация, как составляющая языковой картины мира, представляет собой абстрагирование от момента или периода речи и предполагает временную нелокализованность того, о чем сообщается. Денотат генерализованного субъекта в реальной действительности - это любое лицо или предмет, существующий в окружающем мире, который обобщает и репрезентует всех и каждого члена, реально или потенциально входящего в данное множество. Субьект генерализованной ситуации включает говорящего и адресата и любое другое лицо либо обозначает абстрактное понятие, явление или конкретный предмет с постоянным квалификативным признаком в ситуации временной нелокализованности.

Семантико-прагматическая база генерализации представлена семой собирательности, которая объединяет всех представителей класса и указывает на весь класс в целом, а также семой дистрибутивности, которая распространяет признак или действие на любого представителя класса.

Проблема соотнесения языковых структур с когнитивными в случае генерализованных предложений в английском языке решается за счет заимствования и окказионального использования парадигматических форм других категорий. За исключением местоимения one, являющегося единственным маркером только генерализованной референции, все остальные формальные показатели генерализованности выражают значения других грамматических категорий, наряду с категорией обобщенности. Поскольку формальные средства, представляющие данную категорию, являются неспециализированными, выражаемое ими значение генерализации для них вторично и опреде- ляется как лингвистическим, так и экстралингвистическим контекстом.

Способы выражения субьектности в рамках категории генерализованности могут быть разделены на три категории: местоимения, существительные с артиклем и именные дескрипции.

К местоимениям, употребляющимся в качестве генерализованных субъектов, относятся местоимение one; местоимения anyone, anybody, anything, no one, nobody, nothing; everybody, everyone, everything; you; he; we. Например:

If one takes a closer look, however, it becomes clear that the Chinese leadership is neither pursuing the Marxian ideal of a communist society, in which individuals supposedly live in freedom [3].

Everybody needs access to bathrooms [4].

In recent times we have witnessed, even in the supposedly advanced democracies, the age-old treatment of women as an underclass, as a threat, as a criminal, as a "monster" [5].

Определенный и неопределенный артикли, а также значимое отсутствие артикля среди своих значений имеют значение генерализации. Исчисляемые и неисчисляемые существительные со всеми тремя видами артикля могут выступать в роли генерализованных субъектов в предложении. Например:

Black women have elevated the 'Mama don't take no mess' expression to a form of high art - a narrowing of the eye, a lift of the eyebrow, a tilt of the head. Sometimes there is a sideways arch of the neck, a molasses-slow movement of the jaw that says, without speaking, 'You've got exactly 10 seconds to pick up your feet and run for the hills' [5].

The female is "more like an experienced, well-educated connoisseur evaluating one of the many extraordinary works available to her scrutiny" [5].

A man like Erdogan who, in such a monomaniacal fashion, is so wedded to his own boundless vanity is not anybody who serves his nation well [6].

Под именной дескрипцией понимается сочетание местоимения и существительного, которое выступает в функции субъекта предложения. В данном случае речь идет о сочетании генерализующих местоимений с именем существительным в тех случаях, когда референция осуществляется к целому классу. К именным дескрипциям относятся: местоимения all, any, each, every + существительное. Например:

Any woman who has attempted to occupy any place of influence knows that this process can be a rocky road [5].

\section{Результаты исследования}

Прагматико-целевая установка высказываний с генерализованным субъектом четко соответствует коммуникативно-прагматическим ориентирам публи- 
цистического дискурса. Всем известно, что институты массмедиа играют одну из центральных ролей в конструировании мировоззрения и поведенческих стереотипов современного человека. Именно поэтому в публицистическом дискурсе реализуется функция воздействия (агитации и пропаганды) языка, с которой часто совмещается информативная функция (сообщение нового). В. А. Буряковская пишет, что «если считать, что использование информации напрямую связано с проблемой управления, то средства массовой информации можно рассматривать как особую социально-информационную систему, выполняющую функции ориентации. СМИ создают определенную текстуально-идеологизированную «аудиоиконосферу», в которой живет современный человек и которая служит четкой концептуализации действительности. Именно сфера массовой коммуникации способствует тому, что общество выступает как «генератор социального гипноза», под влиянием которого мы становимся согласованно живущей ассоциацией, именно в СМИ наиболее отчетливо проявляется воздействующая функция языка» [7]. И. В. Жуков, в свою очередь, также выделяет две основные функции дискурса СМИ: ориентирующую и манипулятивную [8].

Поскольку публицистический дискурс освещает широкий спектр вопросов, актуальных для современности и представляющих интерес для общества, информирующая функция этого вида дискурса не вызывает сомнений. По мнению Г. Н. Немец, публицистический дискурс, основанный на системе «методологических операций, направленных на сбор, обработку и распространение социальных знаний в обществе, представляет собой дискурс социоцентрического типа, в котором субъектность колеблется от человека "социального" к человеку "частному". Интерсубъектный характер познания в публицистическом дискурсе определяет сочетание методологических принципов рациональности (поиск истины и истинности в журналистике (публицистике)) и релятивизма (зависимость процесса творческого познания действительности от характера социальных условий)» [9]. Таким образом, прагматической направленностью генерализации, используемой в публицистическом дискурсе, является информирование с помощью переноса общего, характерного для представителей всего класса, на частное, т. е. относящееся к каждому представителю класса, и наоборот. Помимо этого, генерализованные высказывания используются в текстах публицистического дискурса для «информирования о наличии/отсутствии закономерных/ регулярных признаков классов / множеств / определенных групп объектов, а также для выражения субъективно-модальной оценки сообщаемого и для интенсификации высказывания» [10].
В рамках ситуации информирования интенции употребления предложений с генерализованным субъектом могут варьироваться. Приведем классификацию выделенных нами целей употребления предложений с генерализующей семантикой.

\section{I. Описание}

В случае описания в фокусе находятся разные стороны объектов и явлений окружающей действительности:

1) описание характерных признаков;

2) описание типичных действий;

3) описание потенциальных характеристик;

4) описание гиперо-гипонимических отношений;

5) описание по подобию.

При употреблении предложений с генерализованным субъектом для описания у читателя создается впечатление «всеобщей включенности», что «возвращает индивида на новой технологической основе в общность, на этот раз не племенного, а глобального человеческого масштаба» [11]. Рассмотрим приведенные виды описаний подробнее.

\section{1. Описание характерных признаков.}

При описании характерных признаков автор выделяет наиболее существенные признаки и распространяет их на всех представителей описываемого множества.

Homeless people, even though we might look dirty, we don't have showers, we're not smelly animals. We're still human beings [4].

Характеристики, представленные говорящим, не обязательно являются новыми для адресата речи, это может быть неоригинальная характеристика чего-либо, служащая базой для начала или продолжения повествования, например:

Authoritarian regimes consolidate their power at home by providing special benefits to their supporters [12].

Целью описания по характерным признакам является информирование читателей о важных, с точки зрения автора, свойствах и признаках предметов, оно также помогает начать новую мысль или логично и последовательно развить дальнейшее повествование.

\section{2. Описание типичных действий.}

Говорящий выделяет характерные для данного множества субъектов действия и называет или описывает их.

Nuclear weapons serve as protection against attacks from outside, while terror can be used to attack hostile powers on their own territory, especially if border controls are insufficient to detect terrorists gaining entry as refugees [3].

Представляя действия каждого члена множества с помощью генерализованных предложений и типизируя их, автор показывает глобальность называемого явления, что позволяет слушающему/читающему 
осознать предоставленную информацию через призму объединения с другими, принимающими это знание.

After all, other democracies have long put women into the top political post in their country. We women know it is a big deal because - for some reason - men have always, the world over, predominated in such positions of power [5].

В публицистическом дискурсе предложения с генерализованным субъектом могут служить как бы основой, отправным пунктом повествования о каких-либо явлениях или событиях, который помогает автору «строить» дальнейшее повествование для аудитории, уже объединенной общим знанием.

"And we could consign future generations to a life far less agreeable than our own. We could consign our children, our grandchildren, our great grandchildren to a life where there are not only huge movements of populations, huge migrations, but also shortages of food, shortages of water, conflict, caused by climate change. There is absolutely no question this is a reality we must face up to." He added: "I would say that humanity as a whole is about 5-1 down at half time. We've got a long way to go, but we can do it. We have the ability to come back but it's going to take a huge amount of effort" [13].

Понятно, что, представляя мир всех, автор отображает его сквозь призму своего видения реальности. Е. И. Шейгал пишет о том, что при передаче информации коммуниканты предпочтут объединительную функцию информативной [14, с. 127-131]. Так, например, говорящий может описывать свой собственный жизненный опыт, но при этом употреблять генерализованный субъект, подразумевая, что любой человек ведет себя таким же образом в подобной ситуации.

The legacy of slavery is racism. People have unfortunately grown tired of the subject. $<\ldots>$ On the other, we have also borne witness to a sophisticated sanitising of racism - particularly anti-Black racism - as if to admit to suffering racism is to embody fecklessness and a lack of ambition [15].

Эффект причастности, создаваемый предложениями с генерализованным субъектом, используется говорящим для придания передаваемой информации статуса всеобщности, а значит универсальности и значимости для всех.

Reading Huntington's book today, one finds many of his assessments prophetic. In particular, he recognized early on the antagonism of Islamic and Sino cultures toward the West. His prediction that the United States would enter into a struggle with China for global hegemony and that the Islamic world would form a front against the West proves to be on point. Nor can one indignantly reject his statement that "Islam's borders are bloody, and so are its innards" [3].
3. Описание потенциальных характеристик.

В публицистическом дискурсе субъект может быть охарактеризован в соответствии с предположениями автора, которые сделаны на основании имеющихся знаний о субъекте. В этом случае характеристики субъекта предложения являются потенциальными, поскольку могут быть реализованы только при условии наличия подходящих обстоятельств.

Asked whether this meant no agreement had been reached, the spokesperson said: "You would have to ask the French government about whether they want to proceed with the threats they have made" [16].

Генерализованный субъект информирует о том, что при выполнении определенных условий было/ произошло/случилось бы именно так для всех членов множества.

Let's be honest, if we had to spend our life listening and standing next to controversial ex-president Donald Trump, we would also have a similar facial expression to the one that appeared to be sported by Melania during the Major League Baseball (MLB) World Series on Saturday [17].

\section{ний.}

4. Описание гиперо-гипонимических отноше-

Публицистический дискурс характеризуется наличием большого количества дефиниций, определений, понятий, которые «расшифровывает» сам говорящий в своем повествовании. Описание по типу «от единичности к множественности» или «от малого множества к большему множеству» представляет собой вектор от составляющих компонентов, представленных репрезентативным членом, к общему целому, включающему множество членов, подобных тому, который выделяется в данной ситуации.

"It is something that we use as a society to organize ourselves and to organize who gets our resources, opportunities and power," he said. "It's infused into our systems, structures, institutions, practices and our social norms" [18].

\section{5. Описание по подобию.}

В данном случае генерализованный субъект выступает в отношениях подобия/контраста с представителями других классов, описывается его соотношение либо с представителями этого же класса, либо с представителями других классов.

Nuclear weapons serve as protection against attacks from outside, while terror can be used to attack hostile powers on their own territory, especially if border controls are insufficient to detect terrorists gaining entry as refugees [3].

Информирование с помощью описания позволяет презентовать ситуацию с разных ракурсов. Предложения с генерализованным субъектом помогают адресанту достичь поставленной цели, обобщая представленные им сведения и распространяя инфор- 
мацию на всех представителей описываемого множества.

\section{II. Заключение/вывод}

Обобщение представленной ранее информации также относится к способам информирования адресата, и семантический потенциал предложений с генерализованным субъектом эффективно используется для выполнения этой цели.

However some loved the concept of contactless key.

One person wrote: "Love it!!! That would be so convenient."

"I don't get why people are so judgemental about you having a chip in your hand! Literally people put chips in their dogs just in case they go missing," another person said. Someone else replied: "I weirdly love this."

Would you get a chip implanted for the sake of not having to pat yourself down looking for your keys? [19].

Использование генерализованных предложений для подведения итогов в ходе повествования либо в его завершении помогает адресату лучше понять и принять предоставленную информацию, поскольку они имеют необходимые семантические и прагматические характеристики для логичного и последовательного завершения мысли, перехода к финальной точке повествования.

На современном этапе развития общества средства массовой информации, являясь приоритетным источником новых знаний для человека, естественным образом оказывают на него влияние, формируют его мировоззрение и являются призывом к действию. «Картина мира современного человека во многом опосредована теми концептами, стереотипами и установками, которые сообщаются в явной или скрытой форме телевидением, прессой и прочими СМИ» [11]. Под влиянием понимается формирование мнения, убеждений, верований адресата. М. Р. Желтухина рассматривает публицистический дискурс как вид дискурса, имеющего идеологическое и политическое влияние, способствующее формированию общественного мнения и поддержанию социальной общности [20]. А. В. Олянич, в свою очередь, говорит о комплексе «методов, способов и инструментов влияния/воздействия на социум в части формирования вкусов, предпочтений, образа бытия, в глобальном смысле - формирования картины мира» [21, с. $167-$ 201].

Прагматико-целевая установка генерализованных предложений вполне соответствует манипулятивно-директивной функции публицистического дискурca. Инструментарий предложений с генерализованным субъектом, употребляющихся в английском языке, показал, что целевая установка определения ценностного модуса, моделирования образа мысли и действия, и даже руководства к действию может реализовываться в виде следующих вариантов.

\section{1. Ссылка на авторитет.}

Предложения с генерализованным субъектом представляют собой ссылку на обобщенный авторитет, т. е. на компетентное общество/класс/множество. Привлекая авторитетное мнение в целях убеждения адресата, говорящий цитирует источник предоставляемой информации и, таким образом, доказывает истинность информации, поскольку «сведения о происхождении приводимой информации важны для правильного ее понимания и оценки, эффективной работы с материалом» [22].

Ссылка на авторитет в публицистическом дискурce, по мнению А. А. Негрышева, говорит о подмене ответственности, при которой «формально журналист оказывается «над событием», маскируя оценку средствами интертекстуальности» [23]. С. И. Сметанина подтверждает данную точку зрения: «Журналист, получивший свободу слова, не скованный необходимостью идеологически выверять оценки, словно испуганный этим, перекладывает ответственность за публичное слово на другого автора, на другой текст, на читателя, наконец» [24, с. 134].

Ссылки на авторитет помогают адресантам пояснить свою точку зрения словами более авторитетного лица, что оказывает влияние на адресата публицистического дискурса, заставляя его поверить автору, поскольку его слова подкреплены теми, кому нельзя не доверять, например:

Public opinion polls across the world show that trust in government is exceptionally low and that too many politicians are boosting their fortunes and those of their business associates at the public's expense [12].

В ссылках на авторитетные источники может отражаться модальная или эмоциональная оценка ситуации компетентным лицом. Модально-эмоциональная оценка ситуации, высказанная опытным / грамотным / информированным / обличенным полномочиями источником, влияет на оценку ситуации читателем. Она может представлять собой совет, инструкцию по поводу того, как вести себя или что нужно делать в описываемой ситуации, запрет и другие оттенки модальности.

Таким образом, ссылка на авторитет выполняет функцию воздействия на адресата сообщения различными способами, начиная от простого описания ситуации, представленного авторитетным и поэтому заслуживающим доверия лицом до опоры на эмоционально-модальную оценку ситуации.

Прагматические характеристики обобщенных предложений позволяют использовать их в публицистическом дискурсе для ссылки на лицо, являющееся авторитетом для общественного мнения или на культурные и идеологические доминанты, существующие в обществе.

Perpetrators of domestic abuse habitually lie and hide the truth [25]. 
По мнению А. А. Негрышева, интертекстуальность и прецедентная информация, включающая в себя «тексты и символы культуры, составляющие арсенал фоновых знаний представителей того или иного национально-культурного сообщества» [23], позволяют укрепить веру адресата в предоставляемую информацию, более эффективно воздействовать на него, поскольку они «позволяют говорящему использовать в качестве аргумента опору на общекультурные нормы поведения и непреходящие ценности, как присущие данному этническому и социальнокультурному сообществу, так и не имеющие не только временных и пространственных, но также культурных и духовных границ» [10]. Примерами таких предложений публицистического дискурса могут служить следующие.

People say that the bond between a mother and a daughter is unmatched [26].

It is far more likely a victim might seek support from a medical professional, however these records will obviously not be able to be linked to the suitability of a gun licence applicant [25].

Мудрость, накопленная человечеством, цитируется говорящим с целью добиться понимания и поддержки адресата. Опора на опыт человечества вербализуется с помощью предложений с генерализованным субъектом.

You see, among the birds, the male is usually brighter in color and prettier than the female. Of course, that fact usually assumed some kind of male superiority [5].

\section{2. Модальная и эмоциональная оценка ситуа-} ции.

«Оценочность - основной стилеобразующий фактор публицистических материалов - начинает играть свою роль уже на начальной стадии создания текста. Оценочность проявляется в отборе и классификации фактов и явлений действительности, в их описании под определенным углом зрения, в специфических лингвистических средствах. Именно таким образом преобразованную информацию и потребляет читатель» [27]. Оценочность, присвоенная высказыванию автором, направлена на то, чтобы вызвать у адресата ответную реакцию и стимулировать его разделить чувства и эмоции автора.

Анализ материала публицистического дискурса позволил выявить значительное количество видов модальной и эмоциональной оценки ситуации, в которых употребляются генерализованные преложения в английском языке, например, призыв / инструкция к действию, совет, необходимость действия, возможность, способность, твердое намерение, сильное желание/нежелание делать что-либо, протест, критика, упрек, выражение раздражения, запрет и некоторые другие.
Приведем несколько примеров использования генерализованных предложений с целью формирования мировоззрения и побуждения к действию с помощью выражения модальной и эмоциональной оценки ситуации.

You have to think outside the box, but instead we are literally putting them in a box (призыв / инструкция $\kappa$ действию) [28].

One must reckon that the moment when the Kremlin becomes persuaded that Joe Biden will become the next U.S. president must be imminent (необходимость действия) [29].

Users must be able to take their avatars and assets from one part of the Metaverse to another, without it mattering who controls which particular part (необходuмость действия) [30].

We have the means to cripple authoritarian leaders' vast money laundering schemes. We can also stop the flow of hundreds of billions of dollars that currently flow licitly into the treasuries and central banks of governments that are run by officials who refuse to publicly explain just what they do with all this cash (возможность, способность) [12].

The bill also states that teachers can't be compelled "to discuss current events or widely debated and currently controversial issues of public policy or social affairs." However, if they must, they are required to "explore such issues from diverse and contending perspectives without giving deference to any one perspective" (запрет) [18].

Предложения с генерализованным субъектом являются сильным средством воздействия на адресата, поскольку помимо эмоционально-модальной окраски в них присутствует значение распространения на всех возможных адресатов, что убеждает адресата в необходимости действия по примеру всех и вместе со всеми.

\section{Заключение}

Проведенное исследование позволяет сделать вывод о высоком когнитивном потенциале предложений с генерализованным субъектом в английском публицистическом дискурсе.

Хотелось бы подчеркнуть, что семантическая сущность предложений с генерализованным субъектом определяет их прагматическую направленность и ситуации их употребления в публицистическом дискурсе. Генерализованный субъект имеет обобщающую референцию, из этого следует, что характерные черты денотатов обобщающей референции распространяются на любого и каждого члена определенного отдельно взятого класса и являются истинными для них.

С точки зрения когнитивного анализа предложения с генерализованным субъектом отражают такое мироощущение и такой уровень абстракции, при 
котором успешность коммуникации обеспечивается путем ссылки на синтезированный опыт человечества и приобщения к нему. Именно эта особенность предложений с генерализованным субъектом обеспечивает такие прагматические цели высказываний публицистического дискурса, как информирование и воздействие.

\section{ЛИТЕРАТУРА}

1. Кубрякова Е. С. и др. Краткий словарь когнитивных терминов (фрагменты). URL: http://www.infolex.ru/ elibrary_22633501.pdf

2. Маслова В. А. Введение в когнитивную лингвистику : учеб. пособие. М. : Флинта ; Наука, 2004. 296 с.

3. Huntington Reloaded : Reflections on German Escapism. The Globalist, 22.09.21. URL: https://www.theglobalist.com/huntington-reloaded-reflections-on-german-escapism/

4. Everybody needs access to bathrooms. Chicago doesn't provide nearly enough of them. Chicagotribune, 21.10.21. URL: https://www.chicagotribune.com/investigations/ct-chicago-bathroom-access-lacking-20211021rftay5sxgjbkrggvwfvlgvzmvm-htmlstory.html

5. Kamala Harris and the Evolution of the Birds : Worldwide Lessons. The Globalist, 8.11.20. URL: https:// www.theglobalist.com/birds-ornithology-richard-o-prumevolution-darwin-goethe-behavior-feminism-kamala-harris/

6. Erdogan : Everybody's False Friend. The Globalist, 31.01.18. URL: https://www.theglobalist.com/turkey-erdogan-turkey-foreign-policy-nato/

7. Буряковская B. A. Публицистический дискурс как типичный дискурс массовой культуры. URL: http:// izvestia.vspu.ru/files/publics/54/4-7.pdf

8. Жуков И. В. Война в дискурсе современной прессы. URL: http://teneta.rinet.ru/rus/ii/iliazhukov_war.htm

9. Немеи Г. Н. Публицистический дискурс как методологический конструкт. URL: http://vestnik.adygnet. ru/files/2010.4/1008/nemets2010_4.pdf

10. Андиш И. Б. Генерализованные высказывания в публицистическом и художественном дискурсах (на материале современного английского языка). URL: https://pgu.ru/upload/iblock/bea/andish-i.b.pdf

11. Негрышев A. А. О ситуации постмодерна в дискурсивном пространстве СМИ : к проблеме экологии массовой коммуникации. URL: http://www.elcom.ru/ vfnglu/Rus/NetMag/v4/v4 ar15.htm

12. Confronting the Kleptocrats? Contain Their Western(!). The Globalist, 27.10.21. URL: https://www. theglobalist.com/confronting-the-kleptocrats-contain-theirwestern-financial-managers/

13. Boris Johnson warns society could fall at 'terrifying speed' if climate crisis not fixed. Mirror, 29.10.21. URL: https://www.mirror.co.uk/news/politics/boris-johnson-warns-society-could-25332889

14. Шейгал Е. И. Семиотика политического дискурса. Волгоград : Перемена, 2000. 367 с.
15. It's been a turbulent year for race in Britain. So what next? The Guardian, 30.10.21. URL: https://www.theguardian.com/commentisfree/2021/oct/30/race-in-britain-blackhistory-month-equality

16. UK rejects French claim of steps towards agreement over fishing rights row. The Guardian, 30.10.21. URL: https:/www.theguardian.com/politics/2021/oct/31/uk-rejects-french-claim-agreement-fishing-rights-row

17. Melania Trump's facial expression is priceless as she turns away from Donald Trump. Indy100, 30.10.21. URL: https://www.indy100.com/news/melania-trumpsmile-frown-world-series-b1948644

18. Why teachers are struggling with Texas' critical race theory law. Chron, 25.10.21. URL: https:/www.chron.com/ news/houston-texas/article/Why-Texas-critical-race-theory-law-is-confusing-16554399.php

19. Woman 'has chip implanted in her hand' so she can unlock doors and cupboards without keys. Indy100, 12.10.21. URL: https://www.indy100.com/viral/womanchip-implanted-hand-tiktok-b1936726

20. Желтухина М. Р. Специфика влияния вербалики и невербалики масс-медиальной коммуникации на избирательный процесс // Массовая культура на рубеже XX-XXI веков : человек и его дискурс : сб. науч. трудов. М. : Азбуковник, 2003. С. 109-132.

21. Олянич A. В. Гастрономический дискурс в системе массовой коммуникации (семантико-семиотические характеристики) // Массовая культура на рубеже XX-XXI веков : человек и его дискурс : сб. науч. трудов. М. : Азбуковник. 2003. С. 157-160.

22. Болдырева А. А., Кашкин В. Б. Категория авторитетности в научном дискурсе // Язык, коммуникация и социальная среда. 2001. Вып. 1. URL: http://tpl1999. narod.ru/WebLSE2001/ BoldKach.htm

23. Негрышев A. А. Прагматика интертекстуальности в новостном дискурсе СМИ (на материале информационных заметок). URL: http://www.elcom.ru/ vfnglu/Rus/ NetMag/v4/v4_ar08.htm

24. Сметанина С. И. Медиа-текст в системе культуры (динамические процессы в языке и стиле журналистики конца ХХ века). СПб. : Изд-во Михайлова, 2002. $384 \mathrm{c}$.

25. EXCLUSIVE: 'Police gave my abusive ex his guns back despite my pleas - then it was at my chest'. Mirror, 29.10.21. URL: https:/www.mirror.co.uk/news/uk-news/ police-gave-abusive-ex-guns-25325284

26. TikTok swinger stuns platform by revealing that she lets her husband have sex with her mother. Indy100, 05.10.21. URL: https://www.indy100.com/viral/womanchip-implanted-hand-tiktok-b1936726

27. Клушина Н. И. Языковые механизмы формирования оценки в СМИ. URL: http:/www.rusexpert.ru/ magazine/002.htm

28. "Why? Why are you doing this to me?" Chicagotribune, 21.11.21. URL: https://graphics.chicagotribune.com/ illinois-seclusion/ 
29. Putin's Devious Plans If Joe Biden Wins. The Globalist, 23.10.20. URL: https:/www.theglobalist.com/ united-states-2020-presidential-elections-joe-biden-russiavladimir-putin-hybrid-warfare/

30. Wars of the Three Spheres : The West, the East and the Metaverse. The Globalist, 11.10.21. URL: https://www. theglobalist.com/wars-of-the-three-spheres-the-west-theeast-and-the-metaverse/

\section{REFERENCES}

1. Kubryakova E. S., Dem'yankov V. Z., Pankrats YU. G., Luzina L. G. Kratkij slovar' kognitivnykh terminov (fragmenty). Available at: http://www.infolex.ru/elibrary_ 22633501.pdf

2. Maslova V. A. Vvedenie v kognitivnuyu lingvistiku: ucheb. posobie. M.: Flinta; Nauka, 2004. 296 p.

3. Huntington Reloaded: Reflections on German Escapism. The Globalist, 22.09.21. Available at: https:// www.theglobalist.com/huntington-reloaded-reflections-ongerman-escapism/

4. Everybody needs access to bathrooms. Chicago doesn't provide nearly enough of them. Shicagotribune, 21.10.21. Available at: https://www.chicagotribune.com/ investigations/ct-chicago-bathroom-access-lacking20211021-rftay5sxgjbkrggvwfvlgvzmvm-htmlstory.html

5. Kamala Harris and the Evolution of the Birds: Worldwide Lessons. The Globalist, 8.11.20. Available at: https://www.theglobalist.com/birds-ornithology-richard-oprum-evolution-darwin-goethe-behavior-feminism-kamalaharris/

6. Erdogan: Everybody's False Friend. The Globalist, 31.01.18. Available at: https://www.theglobalist.com/turkeyerdogan-turkey-foreign-policy-nato/

7. Buryakovskaya V. A. Publitsisticheskij diskurs kak tipichnyj diskurs massovoj kul'tury URL: http://izvestia. vspu.ru/files/publics/54/4-7.pdf

8. ZHukov I. V. Vojna v diskurse sovremennoj pressy. Available at: http://teneta.rinet.ru/rus/ii/iliazhukov_war.htm

9. Nemets G. N. Publitsisticheskij diskurs kak metodologicheskij konstrukt. Available at: http://vestnik. adygnet.ru/files/2010.4/1008/nemets2010_4.pdf

10. Andish I. B. Generalizovannye vyskazyvaniya v publitsisticheskom i khudozhestvennom diskursakh (na materiale sovremennogo anglijskogo yazyka). Available at: https://pgu.ru/upload/iblock/bea/andish-i.b.pdf

11. Negryshev A. A. O situatsii postmoderna v diskursivnom prostranstve SMI: k probleme ehkologii massovoj kommunikatsii. Available at: http://www.elcom. ru/ vfnglu/Rus/NetMag/v4/v4_ar15.htm

12. Confronting the Kleptocrats? Contain Their Western(!). The Globalist, 27.10.21. Available at: https:// www.theglobalist.com/confronting-the-kleptocrats-containtheir-western-financial-managers/

13. Boris Johnson warns society could fall at 'terrifying speed' if climate crisis not fixed. Mirror, 29.10.21. Available at: https:/www.mirror.co.uk/news/politics/boris-johnsonwarns-society-could-25332889
14. SHejgal E. I. Semiotika politicheskogo diskursa. Volgograd: Peremena, 2000. 367 p.

15. It's been a turbulent year for race in Britain. So what next? The Guardian, 30.10.21. Available at: https://www. theguardian.com/commentisfree/2021/oct/30/race-inbritain-black-history-month-equality

16. UK rejects French claim of steps towards agreement over fishing rights row. The Guardian, 30.10.21. Available at: https://www.theguardian.com/politics/2021/oct/31/ukrejects-french-claim-agreement-fishing-rights-row

17. Melania Trump's facial expression is priceless as she turns away from Donald Trump. Indy100, 30.10.21. Available at: https:/www.indy100.com/news/melaniatrump-smile-frown-world-series-b1948644

18. Why teachers are struggling with Texas' critical race theory law. Chron, 25.10.21. Available at: https://www. chron.com/news/houston-texas/article/Why-Texas-criticalrace-theory-law-is-confusing-16554399.php

19. Woman 'has chip implanted in her hand' so she can unlock doors and cupboards without keys. Indy100, 12.10.21. Available at: https:/www.indy100.com/viral/ woman-chip-implanted-hand-tiktok-b1936726

20. ZHeltukhina M. R. Spetsifika vliyaniya verbaliki i neverbaliki mass-medial'noj kommunikatsii na izbiratel'nyj protsess. In: Massovaya kul'tura na rubezhe XX-XXI vekov: CHelovek i ego diskurs : sb. nauch. tr. M.: Azbukovnik, 2003. Pp. 109-132.

21. Olyanich A. V. Gastronomicheskij diskurs v sisteme massovoj kommunikatsii (semantiko-semioticheskie kharakteristiki). In: Massovaya kul'tura na rubezhe XX-XXI vekov: CHelovek $i$ ego diskurs: sb. nauch. tr. M.: Azbukovnik. 2003. Pp. 157-160.

22. Boldyreva A. A., Kashkin $V$. B. Kategoriya avtoritetnosti v nauchnom diskurse. In: YAzyk, kommunikatsiya i sotsial'naya sreda. 2001. Vyp. 1. Available at: http:// tp11999.narod.ru/WebLSE2001/ BoldKach.htm

23. Negryshev A. A. Pragmatika intertekstual'nosti v novostnom diskurse SMI (na materiale informatsionnykh zametok). Available at: http://www.elcom.ru/ vfnglu/Rus/ NetMag/v4/v4_ar08.htm

24. Smetanina S. I. Media-tekst v sisteme kul'tury (dinamicheskie protsessy v yazyke i stile zhurnalistiki kontsa XX veka). SPb.: Izd-vo Mikhajlova, 2002. 384 p.

25. EXCLUSIVE: 'Police gave my abusive ex his guns back despite my pleas - then it was at my chest'. Mirror, 29.10.21. Available at: https://www.mirror.co.uk/news/uknews/police-gave-abusive-ex-guns-25325284

26. TikTok swinger stuns platform by revealing that she lets her husband have sex with her mother. Indy100, 05.10.21. Available at: https://www.indy 100. com/viral/ woman-chip-implanted-hand-tiktok-b1936726

27. Klushina N. I. YAzykovye mekhanizmy formirovaniya otsenki v SMI. Available at: http://www.rusexpert.ru/ magazine/002.htm

28. "Why? Why are you doing this to me?" Shicagotribune, 21.11.21. Available at: https://graphics.chicagotribune. com/illinois-seclusion/ 
29. Putin's Devious Plans If Joe Biden Wins. The Globalist, 23.10.20. Available at: https://www.theglobalist. com/united-states-2020-presidential-elections-joe-bidenrussia-vladimir-putin-hybrid-warfare/

Воронежский государственный университет Ильичева Н. В., кандидат филологических наук, преподаватель кафедры перевода и профессиональной коммуникации

E-mail: ilichevanv@yandex.ru

Поступила в редакцию 9 октября 2021 г.

Принята к публикаџии 15 октября 2021 г.

\section{Для цитирования:}

Ильичева Н. В. Когнитивный потенциал предложений с генерализованным субьектом в англоязычном публицистическом дискурсе // Вестник Воронежского государственного университета. Серия: Лингвистика и межкультурная коммуникация. 2021. № 4. С. 126-134. DOI: https://doi.org/10.17308/lic.2021.4/3820
30. Wars of the Three Spheres: The West, the East and the MetaverseThe Globalist, 11.10.21. Available at: https:// www.theglobalist.com/wars-of-the-three-spheres-the-westthe-east-and-the-metaverse/

Voronezh State University

Ilyicheva N. V., Candidate of Philology, Associate Professor of the Translation and Professional Communication Department

E-mail: ilichevanv@yandex.ru

Received: 9 Oktober 2021

Accepted: 15 October 2021

\section{For citation:}

Ilyicheva N. V. Cognitive potential of sentences with a generalized subject in English journalistic discourse. Proceedings of Voronezh State University. Series: Linguistics and Intercultural Communication. 2021. No. 4. Pp. 126-134. DOI: https://doi.org/10.17308/lic.2021.4/3820 